\title{
Complex roles of the stroma in the intrinsic resistance to gemcitabine in pancreatic cancer: where we are and where we are going
}

\author{
Chen Liang ${ }^{1,2,3,4}$, Si Shi ${ }^{1,2,3,4}$, Qingcai Meng ${ }^{1,2,3,4}$, Dingkong Liang ${ }^{1,2,3}$, Shunrong Ji ${ }^{1,2,3}$, Bo Zhang ${ }^{1,2,3}$, \\ Yi Qin ${ }^{1,2,3}$, Jin $\mathrm{Xu}^{1,2,3}$, Quanxing $\mathrm{Ni}^{1,2,3}$ and Xianjun $\mathrm{Yu}^{1,2,3}$
}

Pancreatic ductal adenocarcinoma (PDAC) is among the most devastating human malignancies. The poor clinical outcome in PDAC is partly attributed to a growth-permissive tumor microenvironment. In the PDAC microenvironment, the stroma is characterized by the development of extensive fibrosis, with stromal components outnumbering pancreatic cancer cells. Each of the components within the stroma has a distinct role in conferring chemoresistance to PDAC, and intrinsic chemoresistance has further worsened this pessimistic prognosis. The nucleoside analog gemcitabine (GEM) is usually the recommended first-line chemotherapeutic agent for PDAC patients and is given alone or in combination with other agents. The mechanisms of intrinsic resistance to GEM are an active area of ongoing research. This review highlights the important role the complex structure of stroma in PDAC plays in the intrinsic resistance to GEM and discusses whether antistroma therapy improves the efficacy of GEM. The addition of antistroma therapy combined with GEM is expected to be a novel therapeutic strategy with significant survival benefits for PDAC patients.

Experimental \& Molecular Medicine (2017) 49, e406; doi:10.1038/emm.2017.255; published online 1 December 2017

\section{INTRODUCTION}

Pancreatic cancer is among the most devastating of human malignancies and is currently the fourth leading cause of cancerrelated deaths in the United States. ${ }^{1,2}$ Pancreatic ductal adenocarcinoma (PDAC) is the most common form of pancreatic cancer and accounts for $\sim 90 \%$ of all pancreatic tumors. ${ }^{3}$ It is associated with an overall 5 -year survival rate of $<8 \%$, exhibiting the poorest prognosis of all solid tumors. ${ }^{2}$ One of the reasons for this poor prognosis is the high resistance of PDAC to conventional chemotherapy treatments. ${ }^{4,5}$ Although intense research efforts have been made to develop chemotherapy options and patient-targeted therapeutic strategies, there has been no significant improvement in the overall survival (OS). ${ }^{2}$ In addition to overcoming the challenges of chemoresistance, novel therapeutic strategies are desperately needed to improve patient outcomes. ${ }^{2}$

Following the initial success of gemcitabine (GEM) in advanced PDAC, combination therapies with GEM were administered to tackle locally advanced and metastatic disease with limited success. ${ }^{6,7}$ This failure is attributable to many factors, including extrinsic or intrinsic resistance to GEM. ${ }^{8-11}$ Notably,
PDAC is tumor characterized by the development of extensive fibrosis termed desmoplasia, with stromal components outnumbering pancreatic cancer cells. ${ }^{12}$ Thus, PDAC stroma is regarded as a determinant of GEM resistance. Abundant evidence indicates that the stroma plays an important role in extrinsic resistance by impairing GEM delivery (Figure 1); however, the stroma-mediated mechanisms of intrinsic resistance to GEM remain an active area of ongoing investigation. This review focuses on understanding how various components within the stroma are instrumental in mediating intrinsic resistance to GEM and whether antistroma therapies have positive effects on the efficacy of GEM. This research is expected to develop a novel strategy to increase the cytotoxic effects of GEM, eventually achieving a significant survival benefit. The addition of antistroma therapies is expected to increase the cytotoxic effects of GEM, increasing patient survival.

\section{STROMA CONFERS INTRINSIC RESISTANCE TO GEM IN PDAC}

Compared with other malignancies, a cardinal histopathological feature of PDAC is the occurrence of prominent hyperplasia

\footnotetext{
${ }^{1}$ Department of Pancreatic Surgery, Fudan University Shanghai Cancer Center, Shanghai, China; ${ }^{2}$ Department of Oncology, Shanghai Medical College, Fudan University, Shanghai, China and ${ }^{3}$ Pancreatic Cancer Institute, Fudan University, Shanghai, China

${ }^{4}$ These authors contributed equally to this work.

Correspondence: Professor X Yu, Pancreatic Cancer Institute, Fudan University, and Department of Pancreatic and Hepatobiliary Surgery, Fudan University Shanghai Cancer Center, 270 DongAn Road, Shanghai 200032, China.

E-mail: yuxianjun@fudanpci.org

Received 15 May 2017; revised 23 July 2017; accepted 7 August 2017
} 


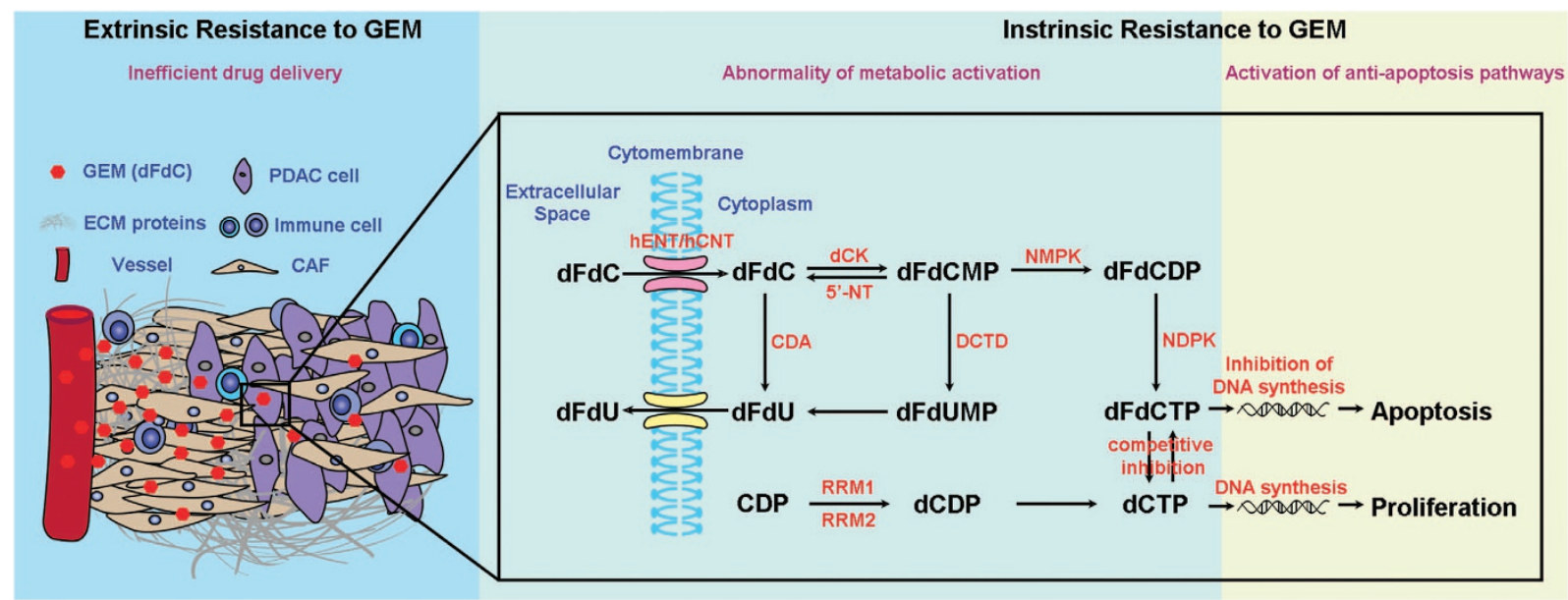

Figure 1 The intrinsic and extrinsic mechanisms of gemcitabine (GEM) resistance. Intrinsic resistance refers to modification of transport mechanisms and the metabolism of the drug and activation of intracellular antiapoptosis pathways. Extrinsic resistance is primarily due to impairment of drug delivery.

of the stroma surrounding the local infiltrated tumor tissues that distorts the normal architecture of pancreatic tissue. ${ }^{13}$ The primary cellular components of stroma are cancer-associated fibroblasts (CAFs), immune cells and endothelial cells, as well as acellular components such as collagens, laminin and cytokines that are stored in the extracellular matrix (ECM). ${ }^{14,15}$ Interactions between the neoplastic and nonneoplastic cells and the acellular matrix have been proposed to stimulate the extensive desmoplastic reaction that is responsible for the main tumor bulk and accounts for up to $90 \%$ of the tumor volume. ${ }^{14,16}$ One gene array analysis indicated that the gene expression pattern in GEM-resistant tumors was enriched in stroma-related pathways. ${ }^{17}$ This experiment highlights the central role of stroma in GEM resistance in PDAC patients. ${ }^{17}$ Stroma confers intrinsic resistance to GEM by mediating the innate or acquired modification of genes involved in GEM metabolism and activation of intracellular antiapoptotic signaling pathways. ${ }^{18,19}$

\section{Stroma affects GEM metabolism in PDAC}

The metabolic availability and activity of GEM toward tumor cells is an important target of stromal interference that leads to intrinsic GEM resistance. Stromal interference of GEM availability and activity against tumor cells highlights the heterogeneous cell populations in the tumor. Certain transporters and metabolic enzymes that process GEM have often been related to GEM resistance in human pancreatic cancer and have therefore been proposed as predictive markers for the response to GEM in a clinical setting. ${ }^{20}$ GEM metabolism is illustrated in Figure 1. Both human equilibrative nucleoside transporter and human concentrative nucleoside transporter transport GEM through the hydrophobic cell membrane. ${ }^{21}$ Once in the cell, GEM (a prodrug; dFdC) is phosphorylated by deoxycytidine kinase to produce $\mathrm{dFdC}$ monophosphate (dFdCMP) and then phosphorylated again by pyrimidine kinases to its active diphosphate and triphosphate derivatives, dFdCDP and dFdCTP. The enzyme opposing deoxycytidine kinase,
5 -nucleotidase, catalyzes the conversion of nucleotides back to the monophosphate form. Moreover, the main mechanism of GEM inactivation is through deamination by cytidine deaminase to difluorodeoxyuridine. Because difluorodeoxyuridine is not a substrate for pyrimidine nucleoside phosphorylases, the drug is degraded and excreted out of the cell. Deamination of dFdCMP to difluorodeoxyuridine by deoxycytidylate deaminase is another inactivation pathway. Importantly, levels of dFdCTP must comprise a sufficient proportion of the cellular pool of dNTPs in order to be efficiently incorporated into DNA, and ribonucleotide reductase plays an essential role in the maintenance of the deoxyribonucleotide pool. ${ }^{20}$ Eventually, the active form of GEM, dFdCTP, is integrated into DNA to impede the synthesis of DNA and exert antitumor effects (Figure 1). Various components within the PDAC stroma can affect the GEM transporters and metabolic enzymes, decreasing the accumulation of the active form of GEM in pancreatic cancer cells. ${ }^{18,22,23}$ Stroma induces epithelial-to-mesenchymal transitions (EMTs) in neoplastic epithelial cells. Notably, EMT results in the suppression of human equilibrative nucleoside transporter/human concentrative nucleoside transporter, ${ }^{24-26}$ inadvertently protecting $\mathrm{EMT}^{+}$ cells from GEM. Thus, the stroma, by impairing GEM metabolism, is a key element in intrinsic GEM resistance. ${ }^{27}$

\section{Stroma activates antiapoptosis pathways to counteract the cytotoxicity of GEM}

The final metabolite, dFdCTP, competes with endogenous dCTP to incorporate in the DNA, leading to the dislodgement of DNA polymerase from the DNA strand. GEM exerts its cytotoxic effects based on DNA damage at a magnitude that cannot be repaired at the cellular level. This masking of GEM by the extra nucleotide eventually induces apoptosis. ${ }^{20,28}$ Resistance to apoptosis has been implicated in the moderate efficiency or failure of a number of anticancer treatments. Thus, another mechanism that contributes to intrinsic GEM resistance involves the activation of DNA damage repair 
Table 1 Clinical trials for antistroma therapy combined with GEM therapy clinical trials that are mentioned in this review

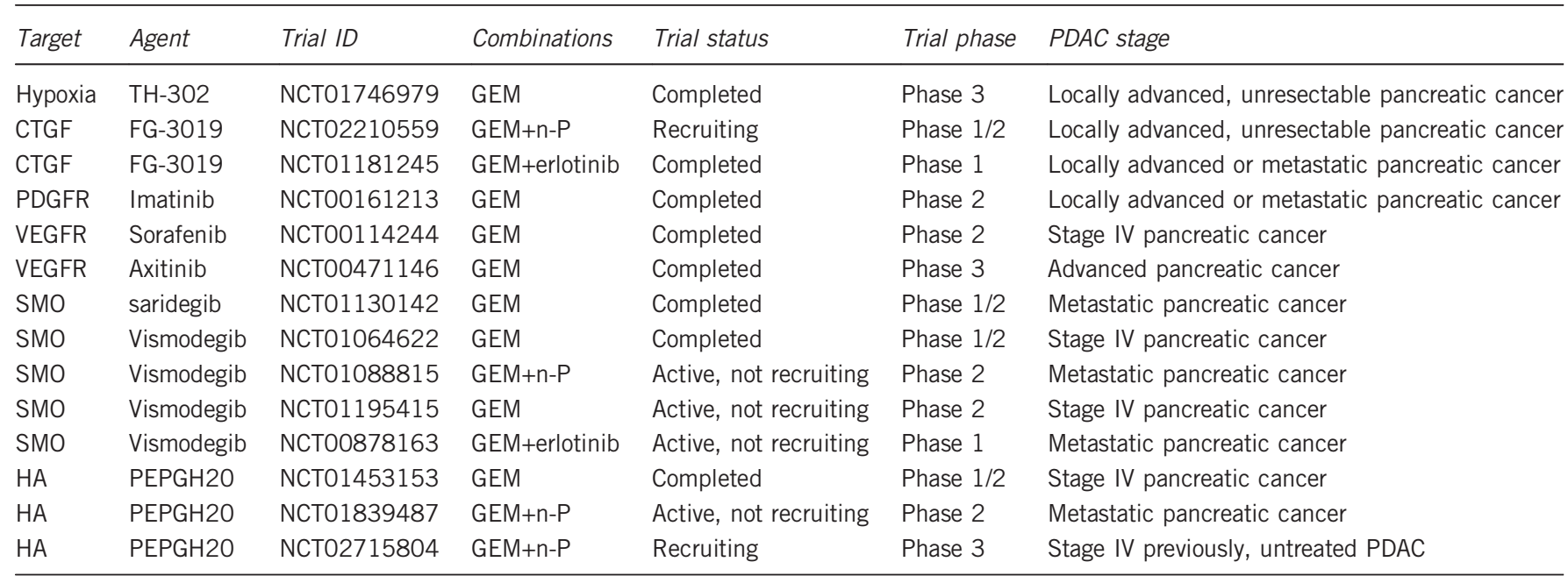

Abbreviations: CTGF, connective tissue growth factor; GEM, gemcitabine; HA, hyaluronan; n-P, nanoparticle albumin-bound-paclitaxel; PDAC, pancreatic ductal adenocarcinoma; PDGFR, platelet-derived growth factor receptor; SMO, smoothened; VEGFR, vascular endothelial growth factor receptor.

pathways and antiapoptosis pathways. This mechanism is deeply rooted in the genetic makeup of PDAC. PDAC harbors high-frequency mutations in major driver genes of cancer, including Kras, CDKN2A, TP53 and SMAD4, numerous lowfrequency driver mutations and regions of hypermutations. ${ }^{29,30}$ These mutations dominate the complex genetic landscape of PDAC and independently hamper the apoptotic process. However, the formation of a complex genetic landscape cannot exist without the influence of the tumor microenvironment. ${ }^{30}$ The stroma affects the expression of various genes and alters the signaling pathways in PDAC cells, including the extracellular signal-regulated kinase (ERK), AKT and signal transducer and activator of transcription 3 (STAT3) pathways. ${ }^{18,31-34}$ Thus, PDAC cells are inherently resistant to GEM because the stroma is the same force that drives malignant transformation as well as GEM resistance ${ }^{20}$ (Figure 1).

\section{MICROENVIRONMENT SUPPORTS PDAC AND COUNTERACTS THE CYTOTOXICITY OF GEM}

\section{A hypoxic microenvironment mediates intrinsic GEM resistance}

Desmoplastic stroma exerts pressure on blood vessels that impairs perfusion and frustrates the excessive metabolic demand for growth, resulting in hypoxic niches with insufficient nutrients. ${ }^{30,35}$ Hypoxia stabilizes hypoxia-inducible factor- $1 \alpha$, a central node that mediates the activation of multiple signaling pathways that may contribute to GEM resistance. ${ }^{24,36}$ Hypoxia-inducible factor- $1 \alpha$ is overexpressed in GEM-resistant PDAC cells and is critically involved in EMT. ${ }^{24,37}$ EMT leads to decreased expression of nucleoside transporters, contributing to decreased sensitivity of PDAC to GEM treatment. ${ }^{27}$ In addition, hypoxia has been shown to maintain stemness that is also associated with GEM resistance. ${ }^{38,39}$ Therefore, areas of hypoxic tumor tissue are more resistant to treatment. ${ }^{40}$ Recently, a hypoxia-activated prodrug, evofosfamide (TH-302), was used to target cancer cells under hypoxic conditions. ${ }^{41}$ It was tested in combination with GEM in a phase III clinical trial for treating patients with advanced pancreatic cancer (MAESTRO, NCT01746979; Table 1). Although there was a trend toward improved OS in patients treated with a combination of the two drugs, it was not a statistically significant improvement. The failure questions the new horizon in the treatment of PDAC: is the role of hypoxia as a potential therapeutic target for the treatment of PDAC ${ }^{42,43}$ However, PDAC is heterogeneous and harbors a heterogeneous microenvironment. Some pancreatic cancers may have a low level of hypoxia, and the inclusion of patients with these tumors might have weakened the statistical power. Thus, patients should be stratified based on the levels of hypoxia in their tumors to reassess the efficacy of combination therapy that is expected to increase OS in patients with high levels of hypoxia.

\section{Microenvironment-induced redox status affects intrinsic GEM resistance}

Microenvironmental stress is involved in cellular metabolism reprogramming, from oxidative phosphorylation to aerobic glycolysis, a remarkable phenomenon called the Warburg effect. ${ }^{35}$ Importantly, glycolytic PDAC cells tend to suppress mitochondrial function. ${ }^{44,45}$ The mitochondrion is an organelle that regulates cell death pathways, not only by controlling intrinsic apoptotic pathways but also by generating reactive oxygen species (ROS). ${ }^{46}$ Excessive production of ROS can cause cellular damage that ultimately leads to cell death. A redox-mediated pathway contributes to the intrinsic resistance of PDAC to GEM. ${ }^{8,47}$ GEM treatment causes intracellular water ionization that produces ROS. ${ }^{8}$ However, the generation of ROS is suppressed in tumor cells by metabolic reprogramming, and the cytotoxic effects are thereby directly reduced, conferring resistance to tumor cells. ${ }^{48,49}$ Thus, cellular redox homeostasis is important to the intrinsic resistance to GEM, and 
modulating ROS generation contributes to the design of drug combinations to overcome the resistance. ${ }^{8}$

\section{An acidic microenvironment mediates the intrinsic resistance to GEM}

The Warburg effect is also an obviously beneficial tradeoff for cancer cells to increase chemoresistance by acidification..$^{50}$ The increase in glycolytic metabolism results in the production of lactate that acidifies the tumor microenvironment. The resulting extracellular acidification, coupled with hypoxia-inducible factor- $1 \alpha$-induced expression of carbonic anhydrases, causes a significant change in the $\mathrm{pH}$ ratio between the intracellular and extracellular environments. This $\mathrm{pH}$ shift decreases the passive absorption of many drugs that would otherwise accumulate at a greater concentration within the cell. ${ }^{51,52}$ An acidic microenvironment can induce EMT that negatively affects GEM uptake. Moreover, acidification also mitigates oxidative stress, ${ }^{53}$ likely providing a defense against GEM-induced ROS that contributes to chemoresistance.

\section{ACELLULAR, STROMAL COMPONENTS INVOLVED IN GEM RESISTANCE}

Type I collagen

PDAC is characterized by a pronounced fibrotic reaction composed primarily of type I collagen. ${ }^{39}$ Type I collagen can affect various behaviors of PDAC, especially metastasis, by maintaining the invasive phenotype of cancer cells or by generating a barrier to invasion. ${ }^{54,55}$ Opposing functions might be performed by different isoforms of type I collagen. ${ }^{54}$ The normal isoform of type I collagen is a heterotrimer that is degraded by CAF-derived collagenases. However, a homotrimeric isoform was found in carcinomas and is normally not present in healthy tissues, suggesting that the homotrimeric protein might enhance the proliferation and migration of cancer cells. ${ }^{56}$ These homotrimers are resistant to all collagenolytic matrix metalloproteinases (MMPs) and are produced by all invasive cancer cell lines, but not by CAFs, thereby comprising a specialized fraction of tumor collagen. Thus, invasive cancer cells may use homotrimers for building MMPresistant invasion highways, facilitating metastasis by directing cancer cells to the vasculature needed for local proliferation and distant spread, whereas surrounding normal heterotrimeric collagens are cleaved..$^{54,56}$

Collagen also contributes to GEM resistance. PDAC cells grown in a three-dimensional collagen microenvironment continue to proliferate in the presence of GEM. ${ }^{18}$ Mechanistically, collagen increases membrane type 1-MMP (MT1MMP) expression in PDAC. ${ }^{25}$ Overexpression of MT1-MMP in the collagen microenvironment increases ERK1/2 phosphorylation and high-mobility group AT-hook 2 (HMGA2) expression and thereby further attenuates the GEM-induced checkpoint arrest to limit the effect of GEM in vitro and in vivo. ${ }^{18}$ Through HMGA2 expression, a three-dimensional collagen microenvironment promotes histone $\mathrm{H} 3 \mathrm{~K} 9$ and H3K27 acetylation and increases histone acetyltransferase expression in PDAC cells to mediate GEM resistance in vitro and in vivo. ${ }^{19}$ Notably, MT1-MMP potentiates integrin signaling only in the three-dimensional microenvironment, thus enhancing PDAC resistance to GEM. ${ }^{57,58}$ Type I collagen induction of EMT is another mechanism of resistance in which pancreatic cancer cells respond to type I collagen by becoming more motile and invasive. Type I collagen binding to receptors upregulates the expression of two mesenchymal markers, Snail and N-cadherin, to further increase the expression of MT1MMP, leading to EMT in PDAC. ${ }^{25,26}$ It is not hard to see the central role of MT1-MMP in collagen-induced GEM resistance, suggesting that targeting MT1-MMP could be a novel approach to sensitizing pancreatic tumors to GEM.

\section{Hyaluronan}

Hyaluronan (HA) is a nonsulfated glycosaminoglycan ECM component produced by HA synthases and degraded by hyaluronidases. ${ }^{59}$ In normal physiological conditions, the amounts of HA in tissues are tightly regulated by a balance between synthesis and degradation. Compared with other malignant tumors in humans, HA content is highest in the desmoplastic stroma of $\mathrm{PDAC}^{60}$ and plays a critical role in a variety of cellular processes, depending on its size and the cell type. ${ }^{59,61}$ A HA-rich microenvironment may promote tumor progression by enhancing cell proliferation, metastasis and angiogenesis. ${ }^{62-64}$ Importantly, strong HA expression is an independent prognostic factor in patients with PDAC. ${ }^{65}$

HA not only affects the malignant behaviors of cancer cells but also protects cancer cells against chemotherapy. HA bound to CD44 increases the phosphorylation of the stem cell marker Nanog. This contributes to an upregulation of the inhibitors of apoptosis proteins and multidrug-resistant protein-1 (MDR1), resulting in antiapoptosis and chemotherapy resistance. ${ }^{66}$ Nevertheless, there is little experimental evidence to support the role of HA in the intrinsic GEM resistance of PDAC. HA induces EMT in PDAC through loss of epithelial E-cadherin and accumulation of cytoplasmic $\beta$-catenin. ${ }^{67}$ However, binding of HA to CD44 activates the Ras/mitogen-activated protein kinase/ERK and phosphatidylinositide 3-kinase-Akt pathways $^{61,68,69}$ that are important for mediating GEM resistance and ensuring cell survival. ${ }^{70,71}$

After taking into account these critical roles of HA in PDAC progression, there has been great interest in developing therapeutic approaches targeting HA. Three different therapeutic approaches have been identified: (1) inhibiting HA synthesis, (2) blocking HA signaling and (3) depleting stromal HA in PDAC to improve chemosensitivity ${ }^{63}$ PEGPH20 is a pegylated hyaluronidase that effectively ablates stromal HA. ${ }^{60,72}$ In addition to being effective in increasing GEM efficacy in a murine PDAC model, ${ }^{72,73}$ PEGPH20 offers more insights for PDAC treatment in future clinical trials. ${ }^{72}$ Some exciting results have been obtained in clinical trials of PEGPH20 (NCT01453153; NCT01839487; NCT02715804), ${ }^{74}$ suggesting that HA ablation may be a promising therapeutic strategy for PDAC with high levels of HA (Table 1). 


\section{Laminin}

Laminin (LN) is another key component of the pancreatic ECM. Cytoplasmic expression of $\mathrm{LN}$ is correlated with a poor prognosis in pancreatic cancer. ${ }^{75}$ In addition, the intrinsic chemoresistance of tumor cells has been shown to be induced by ECM-integrin interactions and is called cell adhesionmediated drug resistance. ${ }^{76} \mathrm{LN}$ is one of the most effective ECM proteins for inducing cell adhesion-mediated drug resistance. ${ }^{77,78}$ Moreover, PDAC cell adhesion to LN with the subsequent activation of signaling pathways contributes to the protection of cancer cells from the cytotoxicity of GEM. ${ }^{10,77,79}$

Focal adhesion kinase (FAK) functions as a critical intracellular molecule in transducing signals from the ECM to cells, and the level of constitutive phosphorylation of FAK at Tyr397 is correlated with the extent of intrinsic GEM resistance in PDAC. ${ }^{34}$ LN-induced FAK phosphorylation is an important event for LN-mediated intrinsic chemoresistance to GEM in PDAC. ${ }^{10} \mathrm{LN}$-induced FAK and AKT phosphorylation increase the levels of survivin expression and Bad phosphorylation at Ser136 that decrease GEM-induced cytotoxicity and apoptosis in PDAC. ${ }^{10}$ FAK is a promising therapeutic target in pancreatic cancer. Targeted therapy against FAK could potentially be used to inhibit the cell-ECM interaction and thus suppress cell adhesion-mediated drug resistance.

\section{Cytokines and chemokines}

Other acellular components of the tumor stroma, soluble cytokines and chemokines, are also central mediators of tumor-stroma crosstalk and are often described as GEM resistance modulators (Table 1). Connective tissue growth factor is overexpressed in PDAC and is currently a target for new therapies. Reportedly, antagonism of connective tissue growth factor with FG-3019, a monoclonal antibody against connective tissue growth factor, enhances GEM sensitivity without altering drug delivery in murine ductal pancreatic cancer ${ }^{80}$ Furthermore, the response to FG-3019 correlates with decreased expression of a previously described promoter of PDAC chemotherapy resistance, the X-linked inhibitor of apoptosis protein. Therefore, alterations in survival cues following targeting of tumor microenvironmental factors may play an important role in PDAC responses to treatment. ${ }^{80}$ FG-3019 has been studied with GEM/erlotinib in localized or metastatic pancreatic cancer and conferred preferable OS rates. ${ }^{81}$ This agent is currently being tested as a neoadjuvant therapy with GEM/nanoparticle albumin-bound paclitaxel (nab-paclitaxel) for locally advanced pancreatic cancer in an attempt to reduce the fibrotic stroma and enhance chemotherapeutic efficacy (NCT02210559). Human PDAC has been reported to overexpress transforming growth factor- $\beta$ that activates the CAFs that are responsible for the formation of the ECM and acts as an immunosuppressor. Therapeutic approaches targeting the transforming growth factor- $\beta$ pathway in PDAC are undergoing clinical trials. ${ }^{14}$

PDAC cells secrete a variety of CXC chemokines into their environment. A highly active axis in the cancer-stroma crosstalk is CXCL12/CXCR4 in PDAC. In vitro, treatment of PDAC cells with CXCL12 activates ERK and AKT signaling and promotes cancer cell resistance to GEM through inhibition of apoptosis. ${ }^{82}$ PDAC cells do not necessarily exploit CXC receptor signaling in an autocrine manner. CXCL1 secretion by PDAC cells induces stromal fibroblasts in a CXCR2dependent manner. Inhibition of CXCR2 also acts synergistically with GEM, resulting in extended survival of animals treated by both a CXCR2 inhibitor and GEM. ${ }^{20,83,84}$ CXCL10, which mediates the crosstalk between pancreatic cancer cells and stellate cells through CXCR2, was recently shown to be correlated with reduced OS. This paracrine signaling pathway confers GEM resistance. ${ }^{85}$

Platelet-derived growth factor is a powerful chemoattractant for pancreatic stellate cells. Unfortunately, although imatinib, an inhibitor of platelet-derived growth factor receptors, improved the efficacy of GEM in preclinical trials, ${ }^{86}$ a recently completed phase II study (NCT00161213) showed no difference in progression-free survival or OS between the imatinib plus GEM and GEM-alone arms of the study. ${ }^{87}$ Targeting stromal-related angiogenic factors such as vascular endothelial growth factor and its receptor also demonstrated no activity when combined with GEM. ${ }^{88-90}$ Poor perfusion and a deficient, nonangiogenic vasculature limits drug delivery and may also help to explain the recent failures of anti-vascular endothelial growth factor strategies in pancreatic cancer.

\section{CELLULAR COMPONENTS WITHIN STROMA INVOLVED IN GEM RESISTANCE}

Cancer-associated fibroblasts

In pathological states, CAFs are activated to transform from the usual quiescent cells to cells with a myofibroblast-like phenotype that express $\alpha$-smooth muscle actin ( $\alpha$-SMA), undergo active proliferation, exhibit enhanced migration and secrete excessive amounts of ECM proteins. ${ }^{24,91,92}$ These $\alpha$-SMApositive $(\alpha-\mathrm{SMA}+)$ CAFs are also called activated pancreatic stellate cells in the pancreas. In addition to secreting insoluble ECM components, CAFs secrete soluble growth, angiogenic and inflammatory factors that engage in cancer and other stromal cell survival and metastatic signaling that promote tumor growth and invasion. ${ }^{91,93,94}$

Importantly, CAFs are innately chemoresistant; they stimulate the same signals in cancer cells as those targeted by therapies, conferring innate resistance. CAFs inhibit cancer cell apoptosis and induce resistance both in vitro and in vivo. ${ }^{91}$ Furthermore, this antiapoptosis mechanism might be involved in the activated status of CAFs. Once the CAFs are induced into quiescence, they play negative roles in cancer cell proliferation and translocation of $\beta$-catenin to the nucleus and can increase cancer cell apoptosis. ${ }^{32,33}$ Activated CAFs can alter tumor morphology, ${ }^{33}$ suggesting that CAFs induce GEM resistance partly through promotion of EMT. CAFs promote EMT in cancer cells by reducing the expression of epithelial markers such as E-cadherin and increasing the expression of mesenchymal markers such as vimentin and Snail. ${ }^{24}$ Moreover, increased Snail expression in cancer cells is a result of CAFderived exosomes. CAFs exposed to GEM dramatically increase 
the release of exosomes that support survival in recipient epithelial cancer cells, and the expression of Snail increases in the exosomes of GEM-treated CAFs. ${ }^{95}$

In addition to the increased release of exosomes, CAFs also secrete proteins that activate survival to promote chemoresistance of pancreatic cancer cells. Conditioned medium from CAFs promotes chemoresistance in PDAC cells and activates the ERK, AKT and STAT3 pathways. ${ }^{31}$ Moreover, CAFs treated with GEM upregulate various inflammatory mediators. This GEM-induced, senescence-associated secretory phenotype is mediated by stress-associated mitogen-activated protein kinase signaling to play tumor-supportive roles in chemotherapytreated CAFs in vitro and in vivo. ${ }^{96}$ This supportive effect is also mediated by paracrine CXCL12 $\alpha /$ CXCR 4 signaling-induced activation of the intracellular FAK/AKT and ERK1/2 signaling pathways in PDAC. ${ }^{97}$ Moreover, CAFs directly support the chemoresistance of PDAC cells by secreting insulin-like growth factor. ${ }^{98}$ As mentioned above, CAFs synthesize abundant insoluble ECM proteins. These ECM proteins provide an ideal microenvironment for pancreatic cancer cells and decrease the cytotoxicity of GEM. ${ }^{77}$ Thus, inhibition of the protein synthesis regulatory pathway represents a promising new therapeutic strategy for improving the chemoresistance triggered by the CAF secretome. ${ }^{99}$

Given the critical roles of CAFs in GEM resistance in PDAC, a therapeutic strategy of inactivation of CAFs, to impair their function, was developed (Table 1). The sonic hedgehog (SHH) pathway is a potent regulator of CAF activation. The SHH pathway is perhaps the most debated mediator of stromainduced chemoresistance in PDAC. In GEM-resistant cell lines, blocking the SHH pathway improves GEM sensitivity. ${ }^{100}$ Moreover, inhibition of the SHH pathway by small-molecule antagonists, such as cyclopamine, vismodegib (GDC-0449), erismodegib and saridegib (IPI-926, a cyclopamine derivative), has shown promising results in multiple preclinical studies. ${ }^{11,101-103}$ Encouraged by these promising results, several clinical trials have been launched using SHH pathway antagonists combined with GEM for PDAC treatment. ${ }^{104}$ Unfortunately, the results have been either negative or equivocal. A phase II placebo-controlled study was conducted in patients with metastatic PDAC to assess the combination treatment of GEM and saridegib (NCT01130142). The clinical trial had to be terminated after the interim data analysis that showed a difference in survival favoring the placebo plus GEM arm due to a higher rate of progressive disease in the saridegib plus GEM arm. The median OS for the saridegib plus GEM arm was $<6$ months, whereas the median OS for the placebo plus GEM arm was $>6$ months. ${ }^{105}$ Similarly, another phase II study did not observe any significant progression-free survival or OS benefit after adding vismodegib to GEM treatment in patients with metastatic PDAC (NCT01064622). ${ }^{106}$ A single-arm phase I/II study using vismodegib in combination with nab-paclitaxel and GEM is currently underway (NCT01088815). An interim analysis estimates a median OS of 10 months for vismodegib in combination with nab-paclitaxel and GEM that is greater than the published historic controls of 8.5 months for GEM plus nab-paclitaxel. ${ }^{107}$ The final results are still awaited and will need to be interpreted further.

The main reason for the conflicting preclinical and clinical results may be the heterogeneity and complex roles of stroma in PDAC. Because the tumor stroma contains many cell types, the number of these cells in the tumor microenvironment and the signal transduction pathways are complex issues. Different components of the stroma have potentially diverse roles. This is better understood in light of new preclinical evidence indicating that inhibition of the $\mathrm{SHH}$ pathway promotes tumor progression in PDAC and at least some stromal constituents can act to restrain tumor progression. ${ }^{108,109}$ Activated stroma with high $\alpha$-SMA expression and low collagen deposition was associated with inferior survival after surgical resection of PDAC. ${ }^{110}$ Interestingly, this activated stroma was dependent on gemcitabine-induced changes in CAFs that were modulated by metronomic chemotherapy. ${ }^{111}$ Moreover, the content of stroma and CAFs may change with different cancer stages. In an attempt to explain these confusing results, SHH pathways were inhibited in three distinct mouse models of Kras-driven PDAC. These preclinical models might only partially recapitulate the complex composition of the PDAC microenvironment, while the stromal desmoplasia was indeed suppressed by $\mathrm{SHH}$ signaling interventions. ${ }^{11,108,109}$ Other reasons for the confusion are the chronic versus acute abrogation of SHH signaling, or the off-target effects of the SHH pathway inhibitors. Thus, inhibition of the SHH pathway has been suggested to act in a synergistic manner with more potent cytotoxic agents, such as nab-paclitaxel. ${ }^{108}$ We need to gain a greater understanding of the involvement of the SHH pathway in PDAC and to determine how this signaling cascade should be targeted.

In addition to inhibiting the activation of CAFs, specifically depleting CAFs is another therapeutic approach to improving the efficiency of GEM in PDAC. In some studies, $\alpha$-SMApositive CAFs and type I collagen were associated with a worse prognosis in patients with PDAC, highlighting the impact of the stromal microenvironment on disease progression and patient survival. ${ }^{112,113}$ However, in the PDAC model with depletion of $\alpha$-SMA+ CAFs, depletion starting at both the early and late stages of pancreatic cancer led to the acceleration of PDAC progression with diminished animal survival, and $\alpha$ SMA+ CAF-depleted tumors suppressed immune surveillance and did not respond to GEM. In PDAC patients, the number of $\alpha$-SMA+ CAFs in their tumors was correlated with survival; specifically, fewer $\alpha$-SMA + CAFs were correlated with a reduced patient survival. ${ }^{14}$ These results suggest that fibrosis associated with $\alpha$-SMA+ CAFs and type I collagen might constitute a protective response from the host rather than offering an oncogenic supportive role, as speculated. ${ }^{114-117}$ A minority of proliferating CAFs in PDAC tumors could aid survival by an $\alpha$-SMA thymidine kinase strategy. An explanation for these inconsistencies is that the origin of CAFs in PDAC is diverse, ${ }^{39,118,119}$ transforming into different subgroups that display various genetic contexts and biofunctions. Together, these studies underscore the need for caution in targeting CAFs in PDAC. ${ }^{39}$ To devise effective treatment 
strategies, instead of merely depleting CAFs, an in-depth understanding of how CAFs react to chemotherapy and how they may contribute to drug resistance is necessary.

\section{Immune cells}

Tumor-infiltrating immune cells can promote chemoresistance and metastatic spread in aggressive tumors. Consequently, the type and quality of immune responses present in the neoplastic stroma are highly predictive of patient outcome in several cancer types. PDAC is characterized as an inflammatory malignancy and an immune suppressive milieu is its bestdescribed hallmark. Cancer immunotherapy generally offers limited clinical benefits without coordinated strategies to mitigate the immunosuppressive nature of the tumor microenvironment. Critical drivers of immune escape in the tumor microenvironment include tumor-associated macrophages and myeloid-derived suppressor cells that not only mediate immune suppression but also promote metastatic dissemination and impart resistance to cytotoxic therapies. Thus, strategies to ablate the effects of these myeloid cell populations may offer great therapeutic potential.

There is abundant intratumoral infiltration of macrophages and most of these tumor-associated macrophages (TAMs) are induced to a M2 phenotype by tumor cell-derived cytokines. Notably, M2-polarized TAMs induce GEM resistance in pancreatic cancer by inhibiting the activation of the caspase-3 pathway during GEM treatment, reducing GEM-induced apoptosis. $22,23,120$ In PDAC models of mice, GEM is more effective in macrophage-depleted mice than in their wild-type counterparts. Similarly, inhibition of monocyte/macrophage trafficking in a transgenic mouse PDAC model that was resistant to GEM alone led to a better response to GEM. ${ }^{121}$ Furthermore, analysis of multiple proteins involved in GEM metabolism revealed that TAMs enhanced the GEM resistance of PDAC by stimulating upregulation of cytidine deaminase. ${ }^{22,23}$ The paracrine effects of TAMs might be mediated by several cytokines released by TAMs. These cytokines, including insulin-like growth factor, interleukin-4 and interleukin-27, activate the relevant pathways in PDAC cells to support acquired resistance to GEM. ${ }^{23,98,122,123}$ TAMs also directly activate the transcription factor STAT3 to enhance the tumor-initiating capacity of pancreatic tumor cells ${ }^{121}$ and STAT3 is involved in GEM sensitivity by downregulation of cytidine deaminase. ${ }^{124}$

\section{CONCLUSIONS}

PDAC is a highly aggressive tumor with poor prognosis that also lacks effective therapeutic regimens compared with other human malignancies. This has encouraged us to improve our understanding of PDAC pathogenesis and biologic function, contributing to the development of a promising strategy for the treatment of PDAC. Desmoplastic stroma is one of the most important histopathological features of PDAC, making it a potential target for PDAC treatment. However, the complex roles of stroma and the failures of clinical trials using antistroma therapy make its future as a PDAC therapy unclear.
Although the complex biochemical cancer-stroma crosstalk within the tumor microenvironment has a critical correlation with intrinsic resistance to GEM, heterogeneity within PDAC contributes significantly to the tumor response to chemotherapy, making this process more complex. Thus, we need to reassess the necessity of antistroma therapy and we should not simply regard it as an indiscriminate depletion of the stroma. More importantly, antistroma therapy combined with GEM should be administered to patients who have been stratified by stromal heterogeneity. An improved resistance to GEM is expected to extend the survival time of PDAC patients given antistroma therapy.

\section{CONFLICT OF INTEREST}

The authors declare no conflict of interest.

\section{ACKNOWLEDGEMENTS}

This study was jointly funded by the National Science Foundation for Distinguished Young Scholars of China (No. 81625016), the National Natural Science Foundation of China (No. 81372651 and 81502031) and the Shanghai Sailing Program (NO. 17YF1402500).

\section{PUBLISHER'S NOTE}

Springer Nature remains neutral with regard to jurisdictional claims in published maps and institutional affiliations.

1 Rahib L, Smith BD, Aizenberg R, Rosenzweig AB, Fleshman JM, Matrisian LM. Projecting cancer incidence and deaths to 2030: the unexpected burden of thyroid, liver, and pancreas cancers in the United States. Cancer Res 2014; 74: 2913-2921.

2 Siegel RL, Miller KD, Jemal A. Cancer statistics, 2017. CA Cancer J Clin 2017; 67: 7-30

3 Ying H, Dey P, Yao W, Kimmelman AC, Draetta GF, Maitra A et al. Genetics and biology of pancreatic ductal adenocarcinoma. Genes Dev 2016; 30: 355-385.

4 Erkan M, Reiser-Erkan C, Michalski CW, Kong B, Esposito I, Friess $\mathrm{H}$ et al. The impact of the activated stroma on pancreatic ductal adenocarcinoma biology and therapy resistance. Curr Mol Med 2012; 12: 288-303.

5 Garrido-Laguna I, Hidalgo M. Pancreatic cancer: from state-of-the-art treatments to promising novel therapies. Nat Rev Clin Oncol 2015; 12 : 319-334.

6 Burris HA 3rd, Moore MJ, Andersen J, Green MR, Rothenberg ML, Modiano MR et al. Improvements in survival and clinical benefit with gemcitabine as first-line therapy for patients with advanced pancreas cancer: a randomized trial. J Clin Oncol 1997; 15: 2403-2413.

7 Sun C, Ansari D, Andersson R, Wu DQ. Does gemcitabine-based combination therapy improve the prognosis of unresectable pancreatic cancer? World J Gastroenterol 2012; 18: 4944-4958.

$8 \mathrm{Ju} \mathrm{HQ}$, Gocho T, Aguilar M, Wu M, Zhuang ZN, Fu J et al. Mechanisms of overcoming intrinsic resistance to gemcitabine in pancreatic ductal adenocarcinoma through the redox modulation. Mol Cancer Ther 2015; 14: 788-798.

9 Koay EJ, Truty MJ, Cristini V, Thomas RM, Chen R, Chatterjee D et al. Transport properties of pancreatic cancer describe gemcitabine delivery and response. J Clin Invest 2014; 124: 1525-1536.

10 Huanwen W, Zhiyong L, Xiaohua S, Xinyu R, Kai W, Tonghua L. Intrinsic chemoresistance to gemcitabine is associated with constitutive and laminin-induced phosphorylation of FAK in pancreatic cancer cell lines. Mol Cancer 2009; 8: 125.

11 Olive KP, Jacobetz MA, Davidson CJ, Gopinathan A, McIntyre D, Honess D et al. Inhibition of Hedgehog signaling enhances delivery of chemotherapy in a mouse model of pancreatic cancer. Science 2009; 324: 1457-1461.

12 Mahadevan D, Von Hoff DD. Tumor-stroma interactions in pancreatic ductal adenocarcinoma. Mol Cancer Ther 2007; 6: 1186-1197. 
13 Apte MV, Park S, Phillips PA, Santucci N, Goldstein D, Kumar RK et al. Desmoplastic reaction in pancreatic cancer: role of pancreatic stellate cells. Pancreas 2004; 29: 179-187.

14 Chu GC, Kimmelman AC, Hezel AF, DePinho RA. Stromal biology of pancreatic cancer. J Cell Biochem 2007; 101: 887-907.

15 Korc M. Pancreatic cancer-associated stroma production. Am J Surg 2007; 194(4 Suppl): S84-S86.

16 Neesse A, Michl P, Frese KK, Feig C, Cook N, Jacobetz MA et al. Stromal biology and therapy in pancreatic cancer. Gut 2011; 60: 861-868.

17 Garrido-Laguna I, Uson M, Rajeshkumar NV, Tan AC, de Oliveira E, Karikari $C$ et al. Tumor engraftment in nude mice and enrichment in stroma- related gene pathways predict poor survival and resistance to gemcitabine in patients with pancreatic cancer. Clin Cancer Res 2011; 17: 5793-5800.

18 Dangi-Garimella S, Krantz SB, Barron MR, Shields MA, Heiferman MJ, Grippo PJ et al. Three-dimensional collagen I promotes gemcitabine resistance in pancreatic cancer through MT1-MMP-mediated expression of HMGA2. Cancer Res 2011; 71: 1019-1028.

19 Dangi-Garimella S, Sahai V, Ebine K, Kumar K, Munshi HG. Threedimensional collagen I promotes gemcitabine resistance in vitro in pancreatic cancer cells through HMGA2-dependent histone acetyltransferase expression. PLOS ONE 2013; 8: e64566.

20 Binenbaum Y, Na'ara S, Gil Z. Gemcitabine resistance in pancreatic ductal adenocarcinoma. Drug Resist Updat 2015; 23: 55-68.

21 Mini E, Nobili S, Caciagli B, Landini I, Mazzei T. Cellular pharmacology of gemcitabine. Ann Oncol 2006; 17 (Suppl 5): v7-12

22 Weizman N, Krelin Y, Shabtay-Orbach A, Amit M, Binenbaum Y, Wong RJ et al. Macrophages mediate gemcitabine resistance of pancreatic adenocarcinoma by upregulating cytidine deaminase. Oncogene 2014; 33: 3812-3819.

23 Amit M, Gil Z. Macrophages increase the resistance of pancreatic adenocarcinoma cells to gemcitabine by upregulating cytidine deaminase. Oncoimmunology 2013; 2: e27231.

24 Kikuta K, Masamune A, Watanabe T, Ariga H, Itoh H, Hamada S et al. Pancreatic stellate cells promote epithelial-mesenchymal transition in pancreatic cancer cells. Biochem Biophys Res Commun 2010; 403 : 380-384.

25 Shields MA, Dangi-Garimella S, Krantz SB, Bentrem DJ, Munshi HG. Pancreatic cancer cells respond to type I collagen by inducing snail expression to promote membrane type 1 matrix metalloproteinasedependent collagen invasion. J Biol Chem 2011; 286: 10495-10504.

26 Huang H, Svoboda RA, Lazenby AJ, Saowapa J, Chaika N, Ding K et al. Up-regulation of $\mathrm{N}$-cadherin by collagen I-activated discoidin domain receptor 1 in pancreatic cancer requires the adaptor molecule Shc1. J Biol Chem 2016; 291: 23208-23223.

27 Zheng X, Carstens JL, Kim J, Scheible M, Kaye J, Sugimoto H et al. Epithelial-to-mesenchymal transition is dispensable for metastasis but induces chemoresistance in pancreatic cancer. Nature 2015; 527: 525-530.

28 Gandhi V, Legha J, Chen F, Hertel LW, Plunkett W. Excision of 2',2'difluorodeoxycytidine (gemcitabine) monophosphate residues from DNA. Cancer Res 1996; 56: 4453-4459.

29 Bardeesy N, DePinho RA. Pancreatic cancer biology and genetics. Nat Rev Cancer 2002; 2: 897-909.

30 Liang C, Qin Y, Zhang B, Ji S, Shi S, Xu W et al. Metabolic plasticity in heterogeneous pancreatic ductal adenocarcinoma. Biochim Biophys Acta 2016; 1866: 177-188.

31 Yamamoto K, Tateishi K, Kudo Y, Hoshikawa M, Tanaka M, Nakatsuka T et al. Stromal remodeling by the BET bromodomain inhibitor JQ1 suppresses the progression of human pancreatic cancer. Oncotarget 2016; 7: 61469-61484.

32 Sherman MH, Yu RT, Engle DD, Ding N, Atkins AR, Tiriac $\mathrm{H}$ et al. Vitamin $D$ receptor-mediated stromal reprogramming suppresses pancreatitis and enhances pancreatic cancer therapy. Cell 2014; 159: 80-93.

33 Froeling FE, Feig C, Chelala C, Dobson R, Mein CE, Tuveson DA et al. Retinoic acid-induced pancreatic stellate cell quiescence reduces paracrine Wnt-beta-catenin signaling to slow tumor progression. Gastroenterology 2011; 141: 1486-1497 1497 el-14.

34 Bouchard V, Harnois C, Demers MJ, Thibodeau S, Laquerre V, Gauthier R et al. B1 integrin/Fak/Src signaling in intestinal epithelial crypt cell survival: integration of complex regulatory mechanisms. Apoptosis 2008; 13: 531-542.
35 Vasseur S, Tomasini R, Tournaire R, Iovanna JL. Hypoxia induced tumor metabolic switch contributes to pancreatic cancer aggressiveness. Cancers (Basel) 2010; 2: 2138-2152.

36 Doktorova H, Hrabeta J, Khalil MA, Eckschlager T. Hypoxia-induced chemoresistance in cancer cells: the role of not only HIF-1. Biomed Pap Med Fac Univ Palacky Olomouc Czech Repub 2015; 159: 166-177.

37 Wang R, Cheng L, Xia J, Wang Z, Wu Q, Wang Z. Gemcitabine resistance is associated with epithelial-mesenchymal transition and induction of HIF-1alpha in pancreatic cancer cells. Curr Cancer Drug Targets 2014; 14: 407-417.

38 Yang MC, Wang HC, Hou YC, Tung HL, Chiu TJ, Shan YS. Blockade of autophagy reduces pancreatic cancer stem cell activity and potentiates the tumoricidal effect of gemcitabine. Mol Cancer 2015; 14: 179.

39 Ozdemir BC, Pentcheva-Hoang T, Carstens JL, Zheng X, Wu CC, Simpson TR et al. Depletion of carcinoma-associated fibroblasts and fibrosis induces immunosuppression and accelerates pancreas cancer with reduced survival. Cancer Cell 2014; 25: 719-734.

40 Bristow RG, Hill RP. Hypoxia and metabolism. Hypoxia, DNA repair and genetic instability. Nat Rev Cancer 2008; 8: 180-192.

41 Lohse I, Rasowski J, Cao P, Pintilie M, Do T, Tsao MS et al. Targeting hypoxic microenvironment of pancreatic xenografts with the hypoxiaactivated prodrug TH-302. Oncotarget 2016; 7: 33571-33580.

42 Erkan M, Kurtoglu M, Kleeff J. The role of hypoxia in pancreatic cancer: a potential therapeutic target? Expert Rev Gastroenterol Hepatol 2016; 10: 301-316.

43 Pourmorteza M, Rahman ZU, Young M. Evofosfamide, a new horizon in the treatment of pancreatic cancer. Anticancer Drugs 2016; 27: $723-725$.

44 Feig C, Gopinathan A, Neesse A, Chan DS, Cook N, Tuveson DA. The pancreas cancer microenvironment. Clin Cancer Res 2012; 18: 4266-4276.

45 Perera RM, Bardeesy N. Pancreatic cancer metabolism: breaking it down to build it back up. Cancer Discov 2015; 5: 1247-1261.

46 Brizel DM, Sibley GS, Prosnitz LR, Scher RL, Dewhirst MW. Tumor hypoxia adversely affects the prognosis of carcinoma of the head and neck. Int J Radiat Oncol Biol Phys 1997; 38: 285-289.

47 Ju HQ, Zhuang ZN, Li H, Tian T, Lu YX, Fan XQ et al. Regulation of the Nampt-mediated NAD salvage pathway and its therapeutic implications in pancreatic cancer. Cancer Lett 2016; 379: 1-11.

48 Zhang H, Gao P, Fukuda R, Kumar G, Krishnamachary B, Zeller KI et al. HIF-1 inhibits mitochondrial biogenesis and cellular respiration in VHLdeficient renal cell carcinoma by repression of C-MYC activity. Cancer Cell 2007; 11: 407-420.

49 Fruehauf JP, Meyskens FL Jr. Reactive oxygen species: a breath of life or death? Clin Cancer Res 2007; 13: 789-794.

50 Kondoh H. Cellular life span and the Warburg effect. Exp Cell Res 2008; 314: 1923-1928.

51 Maftouh M, Avan A, Sciarrillo R, Granchi C, Leon LG, Rani R et al. Synergistic interaction of novel lactate dehydrogenase inhibitors with gemcitabine against pancreatic cancer cells in hypoxia. Br J Cancer 2014; 110: 172-182.

52 Dauer P, Nomura A, Saluja A, Banerjee S. Microenvironment in determining chemo-resistance in pancreatic cancer: Neighborhood matters. Pancreatology 2017; 17: 7-12.

53 Lamonte G, Tang X, Chen JL, Wu J, Ding CK, Keenan MM et al. Acidosis induces reprogramming of cellular metabolism to mitigate oxidative stress. Cancer Metab 2013; 1: 23.

54 Makareeva E, Han S, Vera JC, Sackett DL, Holmbeck K, Phillips CL et al. Carcinomas contain a matrix metalloproteinase-resistant isoform of type I collagen exerting selective support to invasion. Cancer Res 2010; 70: 4366-4374.

55 Yilmaz M, Christofori G. EMT, the cytoskeleton, and cancer cell invasion. Cancer Metastasis Rev 2009; 28: 15-33.

56 Provenzano PP, Eliceiri KW, Campbell JM, Inman DR, White JG, Keely PJ. Collagen reorganization at the tumor-stromal interface facilitates local invasion. BMC Med 2006; 4: 38

57 Duxbury MS, Ito H, Benoit E, Waseem T, Ashley SW, Whang EE.. RNA interference demonstrates a novel role for integrin-linked kinase as a determinant of pancreatic adenocarcinoma cell gemcitabine chemoresistance. Clin Cancer Res 2005; 11: 3433-3438.

58 Shields MA, Dangi-Garimella S, Redig AJ, Munshi HG. Biochemical role of the collagen-rich tumour microenvironment in pancreatic cancer progression. Biochem J 2012; 441: 541-552. 
59 Toole BP. Hyaluronan: from extracellular glue to pericellular cue. Nat Rev Cancer 2004; 4: 528-539.

60 Jacobetz MA, Chan DS, Neesse A, Bapiro TE, Cook N, Frese KK et al. Hyaluronan impairs vascular function and drug delivery in a mouse model of pancreatic cancer. Gut 2013; 62: 112-120.

61 Sato N, Kohi S, Hirata K, Goggins M. Role of hyaluronan in pancreatic cancer biology and therapy: once again in the spotlight. Cancer Sci 2016; 107: 569-575.

62 Itano N, Zhuo L, Kimata K. Impact of the hyaluronan-rich tumor microenvironment on cancer initiation and progression. Cancer Sci 2008; 99: 1720-1725.

63 Sato N, Cheng XB, Kohi S, Koga A, Hirata K. Targeting hyaluronan for the treatment of pancreatic ductal adenocarcinoma. Acta Pharm Sin B 2016; 6: 101-105.

64 Teranishi F, Takahashi N, Gao N, Akamo Y, Takeyama H, Manabe T et al. Phosphoinositide 3-kinase inhibitor (wortmannin) inhibits pancreatic cancer cell motility and migration induced by hyaluronan in vitro and peritoneal metastasis in vivo. Cancer Sci 2009; 100: 770-777.

65 Cheng XB, Sato N, Kohi S, Yamaguchi K. Prognostic impact of hyaluronan and its regulators in pancreatic ductal adenocarcinoma. PLOS ONE 2013; 8: e80765.

66 Bourguignon LY, Spevak CC, Wong G, Xia W, Gilad E. Hyaluronan-CD44 interaction with protein kinase $\mathrm{C}$ (epsilon) promotes oncogenic signaling by the stem cell marker Nanog and the production of microRNA-21, leading to down-regulation of the tumor suppressor protein PDCD4, anti-apoptosis, and chemotherapy resistance in breast tumor cells. J Biol Chem 2009; 284: 26533-26546.

67 Kultti A, Zhao C, Singha NC, Zimmerman S, Osgood RJ, Symons R et al. Accumulation of extracellular hyaluronan by hyaluronan synthase 3 promotes tumor growth and modulates the pancreatic cancer microenvironment. Biomed Res Int 2014; 2014: 817613.

68 Misra S, Hascall VC, Markwald RR, Ghatak S. Interactions between hyaluronan and its receptors (CD44, RHAMM) regulate the activities of inflammation and cancer. Front Immunol 2015; 6: 201.

69 Sohara $Y$, Ishiguro N, Machida K, Kurata H, Thant AA, Senga T et al. Hyaluronan activates cell motility of $\mathrm{v}$-Src-transformed cells via Rasmitogen-activated protein kinase and phosphoinositide 3-kinase-Akt in a tumor-specific manner. Mol Biol Cell 2001; 12: 1859-1868.

70 Datta SR, Dudek H, Tao X, Masters S, Fu H, Gotoh Y et al. Akt phosphorylation of BAD couples survival signals to the cell-intrinsic death machinery. Cell 1997; 91: 231-241.

71 Shi M, He X, Wei W, Wang J, Zhang T, Shen X. Tenascin-C induces resistance to apoptosis in pancreatic cancer cell through activation of ERK/NF-kappaB pathway. Apoptosis 2015; 20: 843-857.

72 Provenzano PP, Cuevas C, Chang AE, Goel VK, Von Hoff DD, Hingorani SR. Enzymatic targeting of the stroma ablates physical barriers to treatment of pancreatic ductal adenocarcinoma. Cancer Cell 2012; 21: 418-429.

73 Bapiro TE, Richards FM, Goldgraben MA, Olive KP, Madhu B, Frese KK et al. A novel method for quantification of gemcitabine and its metabolites 2',2'-difluorodeoxyuridine and gemcitabine triphosphate in tumour tissue by LC-MS/MS: comparison with (19)F NMR spectroscopy. Cancer Chemother Pharmacol 2011; 68: 1243-1253.

74 Hingorani SR, Harris WP, Beck JT, Berdov BA, Wagner SA, Pshevlotsky EM et al. Phase Ib study of PEGylated recombinant human hyaluronidase and gemcitabine in patients with advanced pancreatic cancer. Clin Cancer Res 2016; 22: 2848-2854.

75 Takahashi S, Hasebe T, Oda T, Sasaki S, Kinoshita T, Konishi M et al. Cytoplasmic expression of laminin gamma2 chain correlates with postoperative hepatic metastasis and poor prognosis in patients with pancreatic ductal adenocarcinoma. Cancer 2002; 94: 1894-1901.

76 Damiano JS, Cress AE, Hazlehurst LA, Shtil AA, Dalton WS. Cell adhesion mediated drug resistance (CAM-DR): role of integrins and resistance to apoptosis in human myeloma cell lines. Blood 1999; 93: 1658-1667.

77 Miyamoto H, Murakami T, Tsuchida K, Sugino H, Miyake H, Tashiro S. Tumor-stroma interaction of human pancreatic cancer: acquired resistance to anticancer drugs and proliferation regulation is dependent on extracellular matrix proteins. Pancreas 2004; 28: 38-44.

78 Lee J, Yakubov B, Ivan C, Jones DR, Caperell-Grant A, Fishel M et al. Tissue transglutaminase activates cancer-associated fibroblasts and contributes to gemcitabine resistance in pancreatic cancer. Neoplasia 2016; 18: 689-698.

79 Verma A, Wang H, Manavathi B, Fok JY, Mann AP, Kumar R et al. Increased expression of tissue transglutaminase in pancreatic ductal adenocarcinoma and its implications in drug resistance and metastasis. Cancer Res 2006; 66: 10525-10533.

80 Neesse A, Frese KK, Bapiro TE, Nakagawa T, Sternlicht MD, Seeley TW et al. CTGF antagonism with mAb FG-3019 enhances chemotherapy response without increasing drug delivery in murine ductal pancreas cancer. Proc Natl Acad Sci USA 2013; 110: 12325-12330.

81 Dimou AT, Syrigos KN, Saif MW. Novel agents in the management of pancreatic adenocarcinoma: phase I studies. Highlights from the "2011 ASCO Gastrointestinal Cancers Symposium". San Francisco, CA, USA. January 20-22, 2011. JOP 2011; 12: 114-116.

82 Singh S, Srivastava SK, Bhardwaj A, Owen LB, Singh AP. CXCL12-CXCR4 signalling axis confers gemcitabine resistance to pancreatic cancer cells: a novel target for therapy. Br J Cancer 2010; 103: 1671-1679.

83 McMillin DW, Negri JM, Mitsiades CS. The role of tumour-stromal interactions in modifying drug response: challenges and opportunities. Nat Rev Drug Discov 2013; 12: 217-228.

84 Ijichi H, Chytil A, Gorska AE, Aakre ME, Bierie B, Tada M et al. Inhibiting Cxcr2 disrupts tumor-stromal interactions and improves survival in a mouse model of pancreatic ductal adenocarcinoma. J Clin Invest 2011; 121: 4106-4117.

85 Delitto D, Perez C, Han S, Gonzalo DH, Pham K, Knowlton AE et al. Downstream mediators of the intratumoral interferon response suppress antitumor immunity, induce gemcitabine resistance and associate with poor survival in human pancreatic cancer. Cancer Immunol Immunother 2015; 64: 1553-1563.

86 Hwang RF, Yokoi K, Bucana CD, Tsan R, Killion JJ, Evans DB et al. Inhibition of platelet-derived growth factor receptor phosphorylation by STI571 (Gleevec) reduces growth and metastasis of human pancreatic carcinoma in an orthotopic nude mouse model. Clin Cancer Res 2003; 9: 6534-6544.

87 Moss RA, Moore D, Mulcahy MF, Nahum K, Saraiya B, Eddy S et al. A multi-institutional phase 2 study of imatinib mesylate and gemcitabine for first-line treatment of advanced pancreatic cancer. Gastrointest Cancer Res 2012; 5: 77-83.

88 Kindler HL, Wroblewski K, Wallace JA, Hall MJ, Locker G, Nattam S et al. Gemcitabine plus sorafenib in patients with advanced pancreatic cancer: a phase II trial of the University of Chicago Phase II Consortium. Invest New Drugs 2012; 30: 382-386.

89 Chiorean EG, Coveler AL. Pancreatic cancer: optimizing treatment options, new, and emerging targeted therapies. Drug Des Devel Ther 2015; 9: 3529-3545.

90 Kindler HL, loka T, Richel DJ, Bennouna J, Letourneau R, Okusaka T et al. Axitinib plus gemcitabine versus placebo plus gemcitabine in patients with advanced pancreatic adenocarcinoma: a double-blind randomised phase 3 study. Lancet Oncol 2011; 12: 256-262.

91 Vonlaufen A, Joshi S, Qu C, Phillips PA, Xu Z, Parker NR et al. Pancreatic stellate cells: partners in crime with pancreatic cancer cells. Cancer Res 2008; 68: 2085-2093.

92 Oettle $\mathrm{H}$. Progress in the knowledge and treatment of advanced pancreatic cancer: from benchside to bedside. Cancer Treat Rev 2014; 40: 1039-1047.

93 Hwang RF, Moore T, Arumugam T, Ramachandran V, Amos KD, Rivera A et al. Cancer-associated stromal fibroblasts promote pancreatic tumor progression. Cancer Res 2008; 68: 918-926.

94 Wehr AY, Furth EE, Sangar V, Blair IA, Yu KH. Analysis of the human pancreatic stellate cell secreted proteome. Pancreas 2011; 40: 557-566.

95 Richards KE, Zeleniak AE, Fishel ML, Wu J, Littlepage LE, Hill R. Cancerassociated fibroblast exosomes regulate survival and proliferation of pancreatic cancer cells. Oncogene 2017; 36: 1770-1778.

96 Toste PA, Nguyen AH, Kadera BE, Duong M, Wu N, Gawlas I et al. Chemotherapy-induced inflammatory gene signature and protumorigenic phenotype in pancreatic CAFs via stress-associated MAPK. Mol Cancer Res 2016; 14: 437-447.

97 Zhang H, Wu H, Guan J, Wang L, Ren X, Shi X et al. Paracrine SDF-1alpha signaling mediates the effects of PSCs on GEM chemoresistance through an IL-6 autocrine loop in pancreatic cancer cells. Oncotarget 2015; 6: 3085-3097.

98 Ireland L, Santos A, Ahmed MS, Rainer C, Nielsen SR, Quaranta V et al. Chemoresistance in pancreatic cancer is driven by stroma-derived insulinlike growth factors. Cancer Res 2016; 76: 6851-6863.

99 Duluc C, Moatassim-Billah S, Chalabi-Dchar M, Perraud A, Samain R, Breibach $\mathrm{F}$ et al. Pharmacological targeting of the protein synthesis mTOR/4E-BP1 pathway in cancer-associated fibroblasts abrogates pancreatic tumour chemoresistance. EMBO Mol Med 2015; 7: 735-753. 
100 Chun SG, Zhou W, Yee NS. Combined targeting of histone deacetylases and hedgehog signaling enhances cytoxicity in pancreatic cancer. Cancer Biol Ther 2009; 8: 1328-1339.

101 Kleger A, Perkhofer L, Seufferlein T. Smarter drugs emerging in pancreatic cancer therapy. Ann Oncol 2014; 25: 1260-1270.

102 Yauch RL, Gould SE, Scales SJ, Tang T, Tian H, Ahn CP et al. A paracrine requirement for hedgehog signalling in cancer. Nature 2008; 455: 406-410.

103 Heinemann V, Reni M, Ychou M, Richel DJ, Macarulla T, Ducreux M. Tumour-stroma interactions in pancreatic ductal adenocarcinoma: rationale and current evidence for new therapeutic strategies. Cancer Treat Rev 2014; 40: 118-128.

104 Rosow DE, Liss AS, Strobel O, Fritz S, Bausch D, Valsangkar NP et al. Sonic Hedgehog in pancreatic cancer: from bench to bedside, then back to the bench. Surgery 2012; 152(3 Suppl 1): S19-S32.

105 Anonymous. Infinity reports update from phase 2 study of saridegib plus gemcitabine in patients with metastatic pancreatic cancer http://www. businesswire.com/news/home/20120127005146/en/ Infinity-ReportsUpdate-Phase-2-Study-Saridegib\#U-DoOICSy6w (2012).

106 Catenacci DV, Junttila MR, Karrison T, Bahary N, Horiba MN, Nattam SR et al. Randomized phase $\mathrm{Ib} / \mathrm{ll}$ study of gemcitabine plus placebo or vismodegib, a Hedgehog pathway inhibitor, in patients with metastatic pancreatic cancer. J Clin Oncol 2015; 33: 4284-4292.

107 De Jesus-Acosta A, O'Dwyer PJ, Ramanathan RK, Von Hoff DD, Maitra A, Rasheed $\mathrm{Z}$ et al. A phase II study of vismodegib, a hedgehog ( $\mathrm{Hh}$ ) pathway inhibitor, combined with GEM and nab-paclitaxel (nab-P) in patients (pts) with untreated metastatic pancreatic ductal adenocarcinoma (PDA). J Clin Oncol 2014; 32(Suppl 3): 257.

108 Lee JJ, Perera RM, Wang H, Wu DC, Liu XS, Han S et al. Stromal response to Hedgehog signaling restrains pancreatic cancer progression. Proc Natl Acad Sci USA 2014; 111: E3091-E3100.

109 Rhim AD, Oberstein PE, Thomas DH, Mirek ET, Palermo CF, Sastra SA et al. Stromal elements act to restrain, rather than support, pancreatic ductal adenocarcinoma. Cancer Cell 2014; 25: 735-747.

110 Erkan M, Michalski CW, Rieder S, Reiser-Erkan C, Abiatari I, Kolb A et al. The activated stroma index is a novel and independent prognostic marker in pancreatic ductal adenocarcinoma. Clin Gastroenterol Hepatol 2008; 6: 1155-1161.

111 Chan TS, Hsu CC, Pai VC, Liao WY, Huang SS, Tan KT et al. Metronomic chemotherapy prevents therapy-induced stromal activation and induction of tumor-initiating cells. J Exp Med 2016; 213: 2967-2988.

112 Merika EE, Syrigos KN, Saif MW. Desmoplasia in pancreatic cancer. Can we fight it? Gastroenterol Res Pract 2012; 2012: 781765.

113 Angeli F, Koumakis G, Chen MC, Kumar S, Delinassios JG. Role of stromal fibroblasts in cancer: promoting or impeding? Tumour Biol 2009; 30: 109-120.

114 Wang WQ, Liu L, Xu HX, Luo GP, Chen T, Wu CT et al. Intratumoral alphaSMA enhances the prognostic potency of CD34 associated with maintenance of microvessel integrity in hepatocellular carcinoma and pancreatic cancer. PLOS ONE 2013; 8: e71189.
115 Berchtold S, Grunwald B, Kruger A, Reithmeier A, Hahl T, Cheng T et al. Collagen type $\mathrm{V}$ promotes the malignant phenotype of pancreatic ductal adenocarcinoma. Cancer Lett 2015; 356(2 Pt B): 721-732.

116 Armstrong T, Packham G, Murphy LB, Bateman AC, Conti JA, Fine DR et al. Type I collagen promotes the malignant phenotype of pancreatic ductal adenocarcinoma. Clin Cancer Res 2004; 10: 7427-7437.

117 Omary MB, Lugea A, Lowe AW, Pandol SJ.. The pancreatic stellate cell: a star on the rise in pancreatic diseases. J Clin Invest 2007; 117: 50-59.

118 Phillips P. Pancreatic stellate cells and fibrosisln:Grippo PJ, Munshi HG (eds) Pancreatic Cancer and Tumor Microenvironment. Transworld Research Network: Trivandrum, India, 2012, pp 30-54.

119 Scarlett CJ, Colvin EK, Pinese M, Chang DK, Morey AL, Musgrove EA et al. Recruitment and activation of pancreatic stellate cells from the bone marrow in pancreatic cancer: a model of tumor-host interaction. PLOS ONE 2011; 6: e26088.

120 Pollard JW. Trophic macrophages in development and disease. Nat Rev Immunol 2009; 9: 259-270.

121 Mitchem JB, Brennan DJ, Knolhoff BL, Belt BA, Zhu Y, Sanford DE et al. Targeting tumor-infiltrating macrophages decreases tumor-initiating cells, relieves immunosuppression, and improves chemotherapeutic responses. Cancer Res 2013; 73: 1128-1141.

122 Dijkgraaf EM, Heusinkveld M, Tummers B, Vogelpoel LT, Goedemans R, Jha $\mathrm{V}$ et al. Chemotherapy alters monocyte differentiation to favor generation of cancer-supporting M2 macrophages in the tumor microenvironment. Cancer Res 2013; 73: 2480-2492.

123 Yao L, Wang M, Niu Z, Liu Q, Gao X, Zhou L et al. Interleukin-27 inhibits malignant behaviors of pancreatic cancer cells by targeting $\mathrm{M} 2$ polarized tumor associated macrophages. Cytokine 2017; 89: 194-200.

124 Nagathihalli NS, Castellanos JA, Shi C, Beesetty Y, Reyzer ML, Caprioli R et al. Signal transducer and activator of transcription 3 , mediated remodeling of the tumor microenvironment results in enhanced tumor drug delivery in a mouse model of pancreatic cancer. Gastroenterology 2015; 149: 1932-1943 e9.

(c) (1) () $\ominus$ This work is licensed under a Creative Commons Attribution-NonCommercial-NoDerivs 4.0 International License. The images or other third party material in this article are included in the article's Creative Commons license, unless indicated otherwise in the credit line; if the material is not included under the Creative Commons license, users will need to obtain permission from the license holder to reproduce the material. To view a copy of this license, visit http://creativecommons.org/licenses/by-nc-nd/4.0/

(C) The Author(s) 2017 